\title{
LA CULTURA EMPRENDEDORA PERCIBIDA Y DESEADA EN EL AULA POR LOS ALUMNOS DE EDUCACIÓN SECUNDARIA.
}

\author{
Bianca Dapelo Pellerano \\ Universidad de Playa Ancha \\ bdapelop@yahoo.com \\ Andrés Fernando Avilés Dávila \\ Universidad Complutense de Madrid \\ andresavilesdavila@hotmail.com \\ Francis Edie Flores Castillo \\ Universidad de Playa Ancha \\ francisedie@gmail.com
}

Fecha de Recepción: 2 Marzo 2019

Fecha de Admisión: 30 Abril 2019

\section{RESUMEN}

Se presentan los resultados obtenidos tras la aplicación de los instrumentos expuestos en la comunicación primera de este simposio.

La muestra de estudiantes estuvo formada por 2308 participantes de 18 centros educativos. El $73.6 \%$ de los discentes (1698 estudiantes) pertenecían a institutos públicos, el 23.5\% (542 estudiantes) a centros concertados, y el 2.9\% (68 estudiantes) a centros privados. De estos, 1101 eran hombres (47.7\%) y 1203 mujeres (52.1\%), encontrándose 4 personas sin indicar su género $(0.2 \%)$, El $16.5 \%$ de $1^{\circ}$ ESO; el $14.4 \%$ de $2^{\circ}$ ESO; el $13 \%$ de $3^{\circ}$ ESO; el $20.4 \%$ de $4^{\circ}$ ESO; $21.9 \%$ de $1^{\circ}$ Bachillerato; y el $13.7 \%$ de $2^{\circ}$ Bachillerato.

La edad del alumnado fluctúa de 10 a 18 años, siendo la media 14.84 y la desviación típica 1.77. En cuanto a la procedencia, la muestra estuvo formada por 1922 estudiantes de Asturias (83.3\%), 372 de Castilla y León (16.1\%) y 14 de Cantabria (0.6\%),

La valoración de la cultura emprendedora percibida en las aulas por parte de los alumnos se sitúa en torno a puntuaciones medias tanto en su valoración global como en relación a la finalidad de la educación, procesos de enseñanza, procesos de aprendizaje y escenario educativo. La valoración de la cultura emprendedora deseada en las aulas por parte del alumnado es superior a la percibida, tanto de forma global como en relación a la finalidad de la educación, procesos de enseñanza, procesos de aprendizaje y escenario educativo. Las diferencias detectadas entre la cultura percibida y la cultura deseada en las aulas son significativas. Estos resultados revelan que los alumnos perciben en sus centros unos desempeños docentes en torno a los paradigmas cognitivos y huma- 


\section{LA CULTURA EMPRENDEDORA PERCIBIDA Y DESEADA EN EL AULA POR LOS ALUMNOS DE EDUCACIÓN SECUNDARIA}

nísticos y que apuestan por ello al indicar deseos de mejora en estos desempeños, y estos deseos son al alza y estadísticamente significativos respecto a los percibidos.

Palabras clave: cultura emprendedora percibida; cultura emprendedora deseada; alumnos, educación secundaria.

\section{ABSTRACT}

Perceived and desired entrepreneurial culture in the classroom by secondary school students. The results obtained by the application of the instruments presented in the first communication of this symposium are presented. The student sample consisted of 2308 participants from 18 educational centers. $73.6 \%$ of the students (1698 students) belonged to public institutes, $23.5 \%$ (542 students) to concerted centers, and 2.9\% (68 students) to private centers. Of these students 1101 were men $(47.7 \%)$ and 1203 women (52.1\%), finding 4 people without indicating their gender $(0.2 \%), 16.5 \%$ of $1^{\circ}$ ESO; $14.4 \%$ of $2^{\circ}$ ESO; $13 \%$ of $3^{\circ}$ ESO; $20.4 \%$ of $4^{\circ} \mathrm{ESO} ; 21.9 \%$ of 1 st High School; and $13.7 \%$ of 2 nd High School.

The age of the students varies from 10 to 18 years, being the average 14.84 and the standard deviation 1.77. With respect to the place of origin, the sample consisted of 1922 students from Asturias (83.3\%), 372 from Castilla y León (16.1\%) and 14 from Cantabria (0.6\%).

The assessment of the perceived entrepreneurial culture in the classroom by students is based on average scores both in their overall assessment and in relation to the purpose of education, teaching processes, learning processes and educational setting.

The assessment of the desired entrepreneurial culture in the classrooms by students is superior to that perceived, both globally and in relation to the purpose of education, teaching processes, learning processes and educational setting

Differences detected between the perceived and desired culture in the classrooms are significant. These results reveal that students perceive in their centers some teaching performances closed to cognitive and humanistic paradigms, they bet for it when indicating desires of improvement in these performances, and these desires are upwards and statistically significant with respect to the ones perceived.

Keywords: perceived entrepreneurial culture; desired entrepreneurial culture; students; secondary education

\section{INTRODUCCIÓN}

Tomando como referencia el modelo de análisis de la Cultura organizacional educativa CULTUAULA formulado por Martín del Buey 1991, 1996, 1997, 1998 y 2003, y desarrollado con posterioridad por su equipo de investigación coordinado por la Dra. Martín Palacio , 20102013 en la Universidad Complutense, que ya ha sido presentado en sus líneas básicas en la primera y segunda ponencia de este simposio, en esta ponencia se estudia la percepción que tiene el alumnado de su centro educativo respecto a las creencias en torno a las cuatro dimensiones de análisis propuesta por el modelo cultual, a lo que se añade el estudio de las mejoras posibles que desearía se plasmaran en los desempeños docente de sus profesores.

Es constatable que las preguntas que se formulan en el cuestionario siguen el paradigma cognitivo y humanista de vigente actualidad contraponiéndolo al tradicional y conductual. En consecuencia los resultados que se obtengan estarán valorando el grado de percepción que tienen los alumnos del desempeño docente respecto a estos paradigmas y el grado de deseo de cambio, manifestando implícitamente por ello su conformidad a estos paradigmas.

Interesa conocer el grado de percepción que tienen los alumnos de la actuación de sus profesores respecto a estos paradigmas. De obtener unas puntuaciones bajas se plantearía unas serias 
dudas respecto a la presencia o ausencia en su caso de las creencias implícitas en el profesorado relacionadas con estos paradigmas. Pero eso no sería suficiente si no se pregunta también a los alumnos su deseo de un posible cambio en la misma línea de desempeño docente que propugna actuaciones favorecedoras del desarrollo basado en esos mismos paradigmas.

Si las puntuaciones que se obtienen respecto a los desempeños deseados son altas y las diferencias respecto a las percibidas son significativas, nos estarán confirmado que los desempeños docentes de los profesores en torno a los paradigmas cognitivos y humanistas son consonantes con el deseo de sus alumnos

Esta ponencia se centra únicamente en el estudio de consonancia y disonancias entre las cultura percibida y deseada sin tener en cuenta la existencia de variables moduladoras, condicionantes 0 en su caso determinantes de ellas como sería el género, la edad y el centro. La incidencia de algunas de estas variables son objeto de otras ponencias dentro del simposio.

\section{MÉTODO}

\section{Participantes}

La muestra de estudiantes estuvo formada por 2308 participantes de 18 centros educativos. En la Tabla 1 se presenta la frecuencia sobre el tipo de centro, donde se muestra que el $73.6 \%$ de los discentes (1698 estudiantes) pertenecían a institutos públicos, el 23.5\% (542 estudiantes) pertenecían a centros concertados, y el 2.9\% (68 estudiantes) se encontraban en centros privados.

Tabla 1 Frecuencia de Tipo de centro

\begin{tabular}{lcc}
\hline & & \\
& Frecuencia & Porcentaje \\
\hline Público & 1698 & 73.6 \\
Concertado & 542 & 23.5 \\
Privado & 68 & 2.9 \\
Total & 2308 & 100.0 \\
\hline
\end{tabular}

Los estudiantes participantes de este estudio eran 1101 hombres (47.7\%) y 1203 mujeres $(52.1 \%)$, encontrándose 4 personas que no indicaron su género $(0.2 \%)$, tal y como se muestra en la Tabla 2.

Tabla 2. Frecuencia de género

\begin{tabular}{lcc}
\hline & & \\
& Frecuencia & Porcentaje \\
\hline Hombre & 1101 & 47.7 \\
Mujer & 1203 & 52.1 \\
Perdidos & 4 & 0.2 \\
Total & 2308 & 100.0 \\
\hline
\end{tabular}

En la Tabla 3 se presentan la frecuencia del curso del alumnado, donde se muestra que el $16.5 \%$ era de $1^{\circ}$ ESO; el $14.4 \%$ de $2^{\circ}$ ESO; el $13 \%$ de $3^{\circ}$ ESO; el $20.4 \%$ de $4^{\circ}$ ESO; $21.9 \%$ de $1^{\circ}$ Bachillerato; y el $13.7 \%$ de $2^{\circ}$ Bachillerato. 


\section{LA CULTURA EMPRENDEDORA PERCIBIDA Y DESEADA EN EL AULA POR LOS ALUMNOS DE EDUCACIÓN SECUNDARIA}

\begin{tabular}{lcc}
\multicolumn{3}{c}{ Tabla 3 Frecuencia de curso } \\
\hline & Frecuencia & Porcentaje \\
\hline $1^{\circ}$ ESO & 381 & 16.5 \\
$2^{\circ}$ ESO & 333 & 14.4 \\
$3^{\circ}$ ESO & 301 & 13.0 \\
$4^{\circ}$ ESO & 471 & 20.4 \\
$1^{\circ}$ Bachillerato & 505 & 21.9 \\
$2^{\circ}$ Bachillerato & 317 & 13.7 \\
Total & 2308 & 100.0 \\
\hline
\end{tabular}

Las frecuencias sobre la edad se presentan en la Tabla 4. con un mayor porcentaje de estudiantes entre $15(18,5 \%) y$ años $(22,2 \%)$

Tabla4. Frecuencia de edad

\begin{tabular}{lcc}
\hline & Frecuencia & Porcentaje \\
\hline 10 & 1 & .0 \\
11 & 7 & .3 \\
12 & 310 & 13.4 \\
13 & 293 & 12.7 \\
14 & 315 & 13.6 \\
15 & 426 & 18.5 \\
16 & 513 & 22.2 \\
17 & 383 & 16.6 \\
18 & 49 & 2.1 \\
19 & 6 & .3 \\
20 & 2 & .1 \\
22 & 2 & .1 \\
27 & 1 & .0 \\
Total & 2308 & 100.0 \\
\hline
\end{tabular}

En cuanto a la procedencia del alumnado, la muestra estuvo formada por 1922 estudiantes de Asturias (83.3\%), 372 de Castilla y León (16.1\%) y 14 de Cantabria (0.6\%), Tabla 5.

Tabla 5. Frecuencia de Comunidad Autónoma

\begin{tabular}{lcc}
\hline & Frecuencia & Porcentaje \\
\hline Asturias & 1922 & 83.3 \\
Castilla y León & 372 & 16.1 \\
Cantabria & 14 & .6 \\
Total & 2308 & 100.0 \\
\hline
\end{tabular}




\section{Cuestionario}

Se han empleado los cuestionarios para el análisis de la cultura emprendedora percibida y deseada para alumnos en contextos de educación secundaria (Arnaiz, 2015) presentado en la primera ponencia de este simposio.

En relación a la fiabilidad (índice alfa universalmente aceptado en la investigación psicométricas) de los instrumentos los resultados obtenidos en los cuestionarios son superior al .75: El cuestionario de cultura emprendedora percibida en el alumnado tiene una fiabilidad de: .916; El cuestionario de cultura emprendedora deseada en el alumnado tiene una fiabilidad de: .888

En relación a la validez de estructura se ha obtenido una estructura factorial afín a la planteada en torno a la presencia de cuatro dimensiones o factores la cual a su vez es confirmada mediante el análisis pertinente.

Entrando al detalle nos encontramos que en todos los análisis factoriales exploratorios de la cultura emprendedora percibida y deseada por el alumnado se observa la adecuación de una estructura de un solo factor de las dimensiones Finalidad, Proceso de Enseñanza, Proceso de Aprendizaje y Escenario respectivamente acerca de la cultura emprendedora, teniendo en cuenta el criterio de Timmerman y Lorenzo-Seva (2011) aceptado científicamente.

Por otra parte, se confirma que el modelo cultura se compone de cuatro factores: Finalidad educativa, Proceso de enseñanza, Proceso de aprendizaje y Escenario educativo siguiendo el Modelo 2 que relaciona la cultura organizacional emprendedora con los cuatro factores tanto en cultura percibida como en deseada.

\section{Recogida de datos}

La aplicación de los cuestionarios tuvo lugar en el aula habitual del alumnado dentro del horario escolar. Se realizó de forma colectiva, con una duración aproximada de 15 minutos. Los cuestionarios fueron administrados por personal entrenado para ello y se aseguró el anonimato y la confidencialidad de los datos.

\section{Obtención de datos}

Para el análisis de los datos se empleó el paquete estadístico IBM SPSS Statistics 18.0, el AMOS 18, y el programa Factor 9.3.1 (Lorenzo-Seva y Ferrando, 2015).Para el estudio de diferencias, globales, por factores, y en función del género, edad, curso, tipo de centro se utilizaron las pruebas ANOVA. Los resultados han sido trabajados y elaborados por Arnaiz (2015)

\section{Resultados} do

En la tabla 5 se presenta los descriptivos generales en cultura emprendedora percibida alumna-

Tabla 5. Descriptivos generales en cultura emprendedora percibida alumnado

\begin{tabular}{llc}
\hline & & General \\
\hline Percibida & Media & 77,62 \\
& Desv. Típ. & 15,50 \\
\hline
\end{tabular}

En la tabla 6 se presentan los descriptivos en la cultura emprendedora percibida por el alumnado. 


\section{LA CULTURA EMPRENDEDORA PERCIBIDA Y DESEADA EN EL AULA POR LOS ALUMNOS DE EDUCACIÓN SECUNDARIA}

Tabla 6. Descriptivos por factores en cultura emprendedora percibida alumnado

\begin{tabular}{llcccc}
\hline & & $\begin{array}{c}\text { Finalidad } \\
\text { educativa }\end{array}$ & $\begin{array}{c}\text { Proceso de } \\
\text { enseñanza }\end{array}$ & $\begin{array}{c}\text { Proceso de } \\
\text { aprendizaje }\end{array}$ & \begin{tabular}{c} 
Escenario \\
\hline Percibida
\end{tabular} \\
& Media & 18,71 & 18,90 & 20,29 & 19,70 \\
& Desv. Típ. & 4,70 & 4,17 & 4,33 & 4,213 \\
\hline
\end{tabular}

En la tabla 7 se presenta la media (100.45) y la desviación típica (12.44) en la cultura deseada por el alumnado.

Tabla 7 Descriptivos generales en cultura emprendedora deseada alumnado

\begin{tabular}{llc}
\hline & & General \\
\hline Deseada & Media & 100,45 \\
& Desv. Típ. & 12,44 \\
\hline
\end{tabular}

En la tabla 8 se presentan los descriptivos en la cultura emprendedora deseada por el alumnado.

Tabla 8. Descriptivos por factores en cultura emprendedora deseada alumnado

\begin{tabular}{llcccc}
\hline & & $\begin{array}{c}\text { Finalidad } \\
\text { educativa }\end{array}$ & $\begin{array}{c}\text { Proceso de } \\
\text { enseñanza }\end{array}$ & $\begin{array}{c}\text { Proceso de } \\
\text { aprendizaje }\end{array}$ & Escenario \\
\hline Deseada & Media & 24,89 & 24,70 & 24,95 & 25,89 \\
& Desv. Típ. & 3,84 & 3,57 & 3,62 & 3,53 \\
\hline
\end{tabular}

\section{Diferencias entre la cultura percibida y la deseada en el alumnado}

En primer lugar se comprueba la distribución de las variables mediante la prueba de KolmogrovSmirnov. Los resultados indican que la distribución de todos los factores es no paramétrica, a excepción de la escala global de cultura emprendedora percibida.

En la Tabla 9 se presenta el estudio de diferencias entre la cultura emprendedora percibida y deseada en el alumnado, con las pruebas no paramétricas. Se observa que existen diferencias significativas en todos los factores, siendo la puntuación de la cultura emprendedora deseada estadísticamente superior a la cultura percibida.

Tabla 9. Diferencias cultura percibida y deseada en el alumnado

\begin{tabular}{|c|c|c|c|c|c|}
\hline & $\begin{array}{l}\text { Finalidad } \\
\text { Deseada- } \\
\text { Finalidad } \\
\text { Percibida }\end{array}$ & $\begin{array}{c}\text { Proceso de } \\
\text { Enseñanza } \\
\text { Deseada - } \\
\text { Proceso de } \\
\text { Enseñanza } \\
\text { Percibida } \\
\end{array}$ & $\begin{array}{l}\text { Proceso de } \\
\text { Aprendizaje } \\
\text { Deseada - } \\
\text { Proceso de } \\
\text { Aprendizaje } \\
\text { Percibida } \\
\end{array}$ & $\begin{array}{l}\text { Escenario } \\
\text { Deseado - } \\
\text { Escenario } \\
\text { Percibido }\end{array}$ & $\begin{array}{c}\text { Cultura } \\
\text { Deseada - } \\
\text { Cultura } \\
\text { Percibida }\end{array}$ \\
\hline $\mathrm{Z}$ & $-37,827$ & $-37,863$ & $-34,202$ & $-38,670$ & $-39,840$ \\
\hline $\begin{array}{l}\text { Sig. } \\
\text { Asintót. } \\
\text { (bilateral) }\end{array}$ &, 000 & ,000 & ,000 & ,000 &, 000 \\
\hline
\end{tabular}




\section{Discusión}

Al respecto de la cultura emprendedora percibida en el alumnado de Educación Secundaria, se han encontrado a nivel general puntuaciones medias en torno a 77 siendo el total posible de 120.

La percepción del grupo analizado se sitúa en torno puntuaciones que van del 60 y el 90 sobre un total máximo posible de 120 puntos. Estas puntuaciones nos están indicando que la percepción que tienen los alumnos de cómo son los desempeños en su centro por parte del profesorado en lo referente a la cultura emprendedora presenta algunas carencias que son necesario mejorar, dado que en un grupo de alumnos sus valoraciones están próximas en general en torno a la media aritmética que es de 60 puntos.

No obstante, es oportuno señalar que las valoraciones que hacen los alumnos del quehacer 0 desempeño docente de sus profesores en la línea de actuación que propugnan los paradigmas cognitivos y humanistas no son inferiores en ningún caso a la media. Estos resultados, de alguna forma constituyen una evidencia de en el contexto educativo prevalecen creencias que promueven estos paradigmas. Aunque sin lugar a dudas, desde la perspectiva de los alumnos posiblemente queden varios desempeños con un alcance de desarrollo ligero o bastante mejorable.

Si entramos a analizar las puntuaciones obtenidas en cada una de las dimensiones, observamos que en lo referente a la percepción de la finalidad la puntuación media se sitúa en torno al 18.71, de los procesos de enseñanza en torno a 18.90, los de aprendizaje en torno al 20.20 y lo del escenario del 19.70. con una Siendo en todos los casos la oscilación de la población en torno a la media de más menos 4 puntos. Si se tiene en cuenta que la puntuación máxima es de 30 puntos en cada una de las dimensiones, los datos obtenidos permiten detectar algunas carencias que serían convenientes estudiar y establecer mejoras en el desempeño docente.

En lo referente a la finalidad conviene recordar que el alumno valora el grado en que sus profesores trabajan la autoestima de sus alumnos, su capacidad de resolución de problemas, el análisis de las notas obtenidas, el desarrollo de habilidades de comunicación y la creatividad. Una media de 18.71 sobre una puntuación máxima de 30 en términos relativos no es baja. Está indicando que se trabaja en esta línea, aunque todavía presenta lagunas.

Con respecto a los procesos de enseñanza, la percepción que tienen los alumnos del desempeño de sus profesores en este aspecto es de 18.90. sobre un total máximo de 30 puntos. En ello se está valorando el grado en que el profesorado enseña a buscar información en distintas fuentes (libros, Internet, periódicos...), que en las notas también se tengan en cuenta todas las actividades que se han realizado, que proponga diferentes actividades acerca de lo que explica en clase, por ejemplo, excursiones, proyectos de investigación, debates, exposiciones, etc, que relacione lo que explica en clase con lo que se enseña en otras asignaturas o con temas que el alumnado ya conoce y que motive al alumnado a proponer actividades para trabajar los temas que se explican en clase. Esta puntuación nuevamente en términos relativos no es baja. Indica que el centro se trabaja en la línea del paradigma cognitivo y humanista, aunque se requiere fortalecer.

En relación a la puntuación obtenida respecto a los procesos de aprendizaje que desarrollan sus profesores consistentes en que se motive al alumnado a relacionar situaciones de la vida real con lo explicado en clase, se insista más en la comprensión de lo que se enseña que en la memorización, se valore los trabajos en grupo, se anime al alumnado a exponer trabajos a sus compañeros, se motive al alumnado a expresar con sus propias palabras lo que se explica en clase y se oriente al alumnado sobre cómo preparar su asignatura, es de 20.20. Considerando que la máxima puntuación es de 30, no es una media baja en términos relativos. Estos resultados no dejan de ser alentadores.

Finalmente, la media obtenida respecto a la valoración del escenario en donde se desarrolla la 


\section{LA CULTURA EMPRENDEDORA PERCIBIDA Y DESEADA EN EL AULA POR LOS ALUMNOS DE EDUCACIÓN SECUNDARIA}

actividad docente es de 19,70. Allí el alumno valora aspectos tan variados como la colocación de los muebles en las aulas de tal forma que permita una buena comunicación entre todos los que están en clase (profesorado y alumnado) (por ejemplo con las mesas colocadas en forma de $\mathrm{U}$ ) y que ello permita una buena relación entre todos, que haya espacios adaptados para hacer actividades propuestas por el profesorado como por ejemplo: trabajos con ordenadores, visualización de películas, trabajos en grupos, etc. que en las clases se utilicen nuevas tecnologías como por ejemplo: pizarras digitales, ordenadores, webs... y que se deje tiempo para preparar otras asignaturas. Nuevamente la puntuación media no es baja en términos relativos.

Esta información obtenida respecto a la percepción que tienen los alumnos del desempeño docente del profesorado está indicando el nivel de activación de unos comportamientos respecto a unas cuestiones que se formulan en torno a unos paradigmas concretos. Pero no están indicando el grado de conformidad que presenta el alumno respecto a esos comportamientos y, en consecuencia, a lo que en ellos está implícito.

Por ello en este estudio también se les ha preguntado a los alumnos el grado de conformidad que tiene con este planteamiento. Y esto se hace pidiéndole que valore el grado de deseo de posible mejora de lo que percibe en la realidad.

Ya se ha indicado que de haber puntuaciones más altas y estadísticamente significativas respecto a la realidad percibida nos estaría indicando que el alumnado apuesta por la presencia de comportamientos y creencias subyacentes a los paradigmas cognitivos y humanistas.

En este contexto en relación a la cultura emprendedora deseada en el alumnado de Educación Secundaria, se encontraron puntuaciones medias altas, tanto a nivel general como por factores. En todas las puntuaciones obtenidas se observa que las puntuaciones son más altas entre la percibida y la deseada, y que esas diferencias son estadísticamente significativas.

Al realizar un análisis más minucioso podemos apreciar que, a nivel general, la media de cultura deseada es de 100. 45 sobre un total máximo de 120 puntos. La media percibida fue de 77.62. Existe una diferencia de más de 27 puntos. Esto es indicativo que el alumnado desea un cambio en la línea de los comportamientos que se deducen de los paradigmas cognitivos y humanistas.

Y si entramos en detalle en el análisis de las dimensiones en que se explicita las creencias, se observa que las diferencias entre las puntuaciones percibidas y deseadas en cuanto a finalidad es de 18.71 a 24,89 , en procesos de 18.90 a 24.70 , en procesos de aprendizaje de 20,29 a 24,95 y en relación al escenario de 19,70 a 25,89 .

Nuevamente se observa la tendencia diferenciadora entre la deseada y la percibida, lo que pone en evidencia la expectativa de los alumnos de que sus profesores desarrollen en su quehacer docente comportamientos más coherentes con una cultura emprendedora propugnada por los paradigmas cognitivos y humanista. Resultados que conllevan un singular interés y optimismo.

Estos resultados coinciden con la investigación de Gargallo, Sánchez, y Ferreras, (2010) y los hallados en niveles universitarios por Pizarro, Real y De la Rosa (2011). Martín Palacio, Fernández, y Pizarro (2010); Martín Palacio, Aguado, y Rodríguez (2013). Arnaiz, Di Giusto y Zamorano (2014) y Di Giusto, Castellano y Arnaiz (2014).

\section{CONCLUSIONES}

La valoración de la cultura emprendedora percibida en las aulas por parte de los alumnos se sitúa en torno a puntuaciones medias tanto en su valoración global como en relación a la finalidad de la educación, procesos de enseñanza, procesos de aprendizaje y escenario educativo.

La valoración de la cultura emprendedora deseada en las aulas por parte del alumnado se sitúa por encima de la media tanto de forma global como en relación a la finalidad de la educación, pro- 
cesos de enseñanza, procesos de aprendizaje y escenario educativo. En consecuencia, se confirma la hipótesis de que los alumnos desean en sus aulas el desarrollo de una cultura emprendedora

La existencia de diferencias entre la cultura percibida y la cultura deseada en las aulas por parte de los alumnos, en cuanto a la finalidad educativa, procesos de enseñanza, procesos de aprendizaje y escenario educativo, son significativamente altas, a favor de la deseada.

Todo ello es indicativo que los alumnos perciben en sus centros unos desempeños docentes en torno a los paradigmas cognitivos y humanísticos y que apuestan por ello al indicar deseos de mejora en estos desempeños y estos deseos son al alza y estadísticamente significativos respecto a los percibidos.

\section{REFERENCIAS BIBLIOGRÁFICAS}

Arnaiz, Garcia,2015: La cultura emprendedora en la educación secundaria. Tesis doctoral. Facultad de Psicología. Universidad de Oviedo.

Arnaiz, A., Di Giusto, C. y Zamorano, M. (2014). Cultura organizacional en la Universidad de Burgos. Estudio diferencia por ramas educativas. Actas del Congreso Internacional Docencia Universitaria e Innovación. Tarragona.

Arnaiz, A., Pizarro, J.P. y Martín del Buey, F. (2012). Análisis de la cultura organizacional percibida y paradigma por alumnos en contextos universitarios: estudio piloto de la facultad de psicología de la Universidad de Oviedo. Actas de VII congreso Iberoamericano de Docencia Universitaria. Facultad de Psicologia e de Ciências da Educação da Universidad do Porto, Porto, Portugal.

Di Giusto, C., Castellanos, S. y Arnaiz, A. (2014). Cultura organizacional en la Universidad de Burgos. Estudio diferenciado por género. Actas del Congreso Internacional Docencia Universitaria e Innovación. Tarragona.

Gargallo, B., Sánchez, F. y Ferreras, A. (2010). Estilos docentes de los profesores universitarios. La percepción de los alumnos de los buenos profesores. Revista Iberoamericana de Educación, 51(4), 1-16.

Martín del Buey, F. (1991). Modelo de análisis de presunciones culturales en el aula. Actas del I Congreso Internacional de Psicología y educación (pp. 168-170). Madrid.

Martín del Buey, F. (1996). El rol del profesor en el umbral del siglo XXI. Revista Magister, 14, 205215.

Martín del Buey, F. (1997). Modelos implícitos en la reforma educativa. Revista Magister, 15, 90-97.

Martín del Buey, F. (1998). Psicología de las culturas. Actas del Congreso internacional sobre el discurso artístico Norte y Sur. Volumen II, (pp. 415-438). Oviedo.

Martín del Buey, F. (2003). La cultura del chip en el aula: del conflicto a la seducción sin perder identidad. X Congreso internacional sobre el discurso artístico: "El discurso visual". Volumen II, (pp. 81-97). Oviedo.

Martín Palacio, M.E., Fernández, A. y Pizarro, J.P. (2010). De la cultura del espectáculo al saber académico sin perecer en el intento: una experiencia de empleo de la producción fílmica en la universidad. Internacional Journal of Developmental and Educational Psychology (INFAD Revista de Psicología.Psicología Positiva, nuevas tecnologías y realidad actual), 4, 229-236

Martín Palacio, M.E., Aguado, J.J. y Rodríguez, E. (2013). Cultura percibida y deseada en contextos educativos universitarios. En J.J. Gázquez Linares, M.C. Pérez Fuentes y M.M. Molero Jurado (Comps.), La Convivencia Escolar: Un acercamiento multidisciplinar (pp. 69-74). Almería: ASUNIVEP.

Pizarro, I., Real, J.C. y De la Rosa, M.D. (2011). La incidencia del capital humano y la cultura emprendedora en la innovación. Cuadernos de Economía y Dirección de la Empresa, 14, 139150. 
